\title{
Cohesive traction-separation relations for tearing of ductile plates with randomly distributed void nucleation sites
}

Andersen, R. G. ; Tekolu, C.; Nielsen, K. L.

Published in:

International Journal of Fracture

Link to article, DOI:

$10.1007 / \mathrm{s} 10704-020-00454-2$

Publication date:

2020

Document Version

Peer reviewed version

Link back to DTU Orbit

Citation (APA):

Andersen, R. G., Tekolu, C., \& Nielsen, K. L. (2020). Cohesive traction-separation relations for tearing of ductile plates with randomly distributed void nucleation sites. International Journal of Fracture, 224, 187-198.

https://doi.org/10.1007/s10704-020-00454-2

\section{General rights}

Copyright and moral rights for the publications made accessible in the public portal are retained by the authors and/or other copyright owners and it is a condition of accessing publications that users recognise and abide by the legal requirements associated with these rights.

- Users may download and print one copy of any publication from the public portal for the purpose of private study or research.

- You may not further distribute the material or use it for any profit-making activity or commercial gain

- You may freely distribute the URL identifying the publication in the public portal 


\title{
Cohesive traction-separation relations for tearing of ductile plates with randomly distributed void nucleation sites
}

\author{
R.G. Andersen • C. Tekoğlu • K.L. Nielsen
}

Received: date / Accepted: date

\begin{abstract}
Cohesive zone traction-separation relations, and the related phenomenological parameters, for steadystate ductile plate tearing, are strongly tied to the micromechanics governing the void nucleation and growth process leading to localized deformation and micro-crack formation. The effects of such local variations on the damage evolution and cohesive zone parameters, respectively, are brought out in this study. A 2D plane strain model setup, first considered in Nielsen and Hutchinson (2012) [Cohesive traction-separation laws for testing of ductile metal plates, Int. J. Impact Eng., 48:15$23]$, is adopted, but here by discretely modeling a finite number of finite-size void nucleation sites distributed randomly in the plate material. It is found that the heterogeneous material conditions, resulting from the nucleation process, strongly affect the localization of damage and fracture, which influence the cohesive energy. By considering a number of realizations of the random distribution for each material configuration, it is concluded that: i) the peak force in the cohesive tractionseparation relation is, essentially, unaffected by the heterogeneity coming into play through the damage-related microstructure, while ii) the cohesive energy decreases
\end{abstract}

R.G. Andersen (凶) (ORCID: 0000-0003-1107-6257)

Solids Mechanics, Department of Mechanical Engineering,

Technical University of Denmark

DK-2800 Kgs. Lyngby, Denmark

Tel.: +4545251960

E-mail: rgra@mek.dtu.dk

C. Tekoğlu (ORCID: 0000-0001-7383-3909)

Department of Mechanical Engineering

TOBB University of Economics and Technology

Sög̈ütözü, Ankara, 06560, Turkey

K.L. Nielsen (ORCID: 0000-0002-0502-8008)

Solids Mechanics, Department of Mechanical Engineering,

Technical University of Denmark

DK-2800 Kgs. Lyngby, Denmark when either increasing the number or the size of the nucleation sites. The cohesive energy is found to be in the range of those previously reported for homogeneous materials, but a direct comparison should be made with caution. The results imply that care should be taken if the actual material configuration diverges from a homogeneous microstructure such as when considering very thin plates and for plates with a few void nucleation - sites.

Keywords Ductile failure - Gurson model · Micromechanics · Size effect $\cdot$ Finite element method

\section{Introduction}

A homogenized continuum finite element model must be approached with care when imperfections in a material have a significant influence on the response of the structure. The heterogeneity originating from the discrete events of void nucleation and growth to coalescence is no exception to this. In a recent study, Srivastava et al. (2017) showed that discrete void nucleation events can alter the crack growth path in ductile metals. They engineered the damage-related microstructure of a metal plate, within a micro-mechanics based numerical framework, and obtained a much-improved fracture toughness of the engineered material by tailoring the crack path (see a related study in Osovski et al., 2019). Srivastava et al. (2017) exploited the fact that the ductile crack tip follows the path where localization is the easiest (i.e., requiring the least amount of energy). A similar approach to increasing the fracture toughness for $\mathrm{Al} / \mathrm{NiTi}$ composites was taken by Zhao et al. (2019), where a local pre-stress state was deliberately introduced near the inclusions within the matrix 
to divert the propagating tearing crack. This interaction between nucleation sites is rarely dealt with, and the local (at times intense) variation in stress/strain is homogenized by adopting a continuum framework such as the classical Gurson model (Gurson, 1977; Gologanu et al., 1997). Nonetheless, the class of Gurson type models facilitates a micro-mechanics based approach to link the underlying damage event to the failure of a structure on the engineering scale.

In recent years, cohesive traction-separation relations suited for ductile plate tearing have been based on the predictions of the Gurson model by homogenizing the material. These phenomenological models have further been used to predict the response of structures on the engineering scale (see Woelke et al., 2015). In a first study, Nielsen and Hutchinson (2012) considered a mode I crack propagating under steady-state ductile plate tearing and developed a simplified 2D plane strain framework that allows the key parameters for the cohesive zone relation to be extracted. The cohesive traction-separation relation must engage at peak traction, related to the Considère condition when the plate cross-section starts to thin and the post-localization has to be accounted for through the energy going into the traction-separation relation. The appearance of the traction-separation relation can, in practice, be approximated by a tri-linear relation with an initial slope of nearly infinite stiffness. Then, a subsequent nearly flat part connects the peak traction to a point identified as the onset of secondary (shear) localization within the thinning region (failure occurs in a slant manner). Finally, a decreasing part that governs the intensification of the secondary (shear) localization and the associated loss of load-carrying capacity (fracture) (see the discussion in Cornec et al., 2003). The 2D setup was later used in Andersen et al. (2018) to investigate the effect of mode mixity within a specialized numerical model that allows for combinations of mode I/II and mode I/III. A minor change to the peak traction was observed when increasing the mode mixity, whereas the relative displacement at the point of secondary (shear) localization and the cohesive energy depend on both the load case and the mixity ratio. The cohesive traction-separation relations extracted from 2D plane strain Gurson calculations have recently been compared to full $3 \mathrm{D}$ simulations accounting for the crack propagation from initiation to steady-state (see Andersen et al., 2019). A near-perfect agreement between the 2D and 3D simulation results was obtained at steady-state, cementing the validity of the $2 \mathrm{D}$ approach to a rather complex ductile fracture problem.

Random distributions of void nucleation sites within the fracture process zone largely determine both the fracture toughness and the roughness of the fracture surfaces (Srivastava et al., 2014). In fact, it is easy to imagine that randomly distributed nucleating voids, which affect the micro-mechanics of failure, can also trigger the transition between different fracture surface morphologies (see Pardoen et al., 2004; Noell et al., 2018, for comprehensive reviews). For example, a substantial amount of nearby-lying nucleation sites give rise to multiple void interaction, while a few widespread nucleation sites will link-up through the void-by-void mechanism (Tvergaard and Hutchinson, 2002). The 2D plane strain setup considered in Nielsen and Hutchinson (2012) was further exploited in Tekoğlu and Nielsen (2019) to demonstrate the transition between fracture surface morphologies as the size and the number of void nucleation sites change. Here, by considering various realizations of a random distribution of the nucleation sites. The fracture surface morphology is largely tied to the type of interaction between the void nucleation sites. Essentially, the morphology is determined by the overall strain hardening capacity of the plate material, taking into account the number and size of the void nucleation sites, and not only the strain hardening of the matrix material. Tekoğlu and Nielsen (2019) have investigated a large spectrum of materials spanning both plates with a high overall hardening capacity (with few small nucleation sites), where cracks predominantly propagate in a cup-cup morphology, and plates with a low overall hardening capacity (with many large nucleation sites) that typically display slant crack propagation. The present work takes up the study in Tekoğlu and Nielsen (2019) with the aim to reveal the effects of introducing discrete void nucleation sites in thin plate tearing under mode I on the cohesive energy and peak force, and to relate the governing micro-mechanisms to the tearing energy. The numerical framework from Tekoğlu and Nielsen (2019) is adopted to extract cohesive zone relations for steady-state ductile tearing in line with Nielsen and Hutchinson (2012).

The 2D approximation of the mode I tearing problem is outlined in Section 2, along with the details on how the cohesive traction-separation relation is identified. The constitutive relations and finite element framework are presented in Section 3, and the results for the energy dissipation, the peak force, and the appearance of the traction-separation relation are given in Section 4. Section 5 summarizes the main findings of this study. 


\section{Problem formulation and cohesive zone identification}

\subsection{Problem formulation}

Steady-state plate tearing under far-field mode I loading of a homogeneous, though porous, metal plate was first addressed within a 2D plane strain setup in Nielsen and Hutchinson (2012). Here, following the line of argument that as plastic flow localization and thinning in the plate cross-section takes place far ahead of the leading crack tip (Considère-like thinning), the material above and below the thinning region unloads. This enforces a plane strain condition for the cross-sections in the fracture process zone with surface normal vectors along the crack growth direction (an assumption later validated by Andersen et al., 2019, in 3D simulations). Nielsen and Hutchinson (2012) used the 2D model setup to extract information about the peak force at the onset of the thinning (the primary localization), the energy going into developing the thinning and the fracture, as well as knowledge on the onset of the (secondary) shear localization. The present study adopts a similar 2D plane strain framework but allows for discrete modeling of randomly distributed void nucleation sites to bring out the effect of material heterogeneity. The setup is an approximation as the tearing process for such heterogeneous, or dual-phase like, metal plates never settles into steady-state at the very tip. However, the $2 \mathrm{D}$ model setup is expected to give a first indication of the trends for the key parameters going into cohesive traction-separation relations which could be obtained if the same comprehensive parameter study was conducted in a $3 \mathrm{D}$ setting. Figure 1 (a) presents a schematic of the plate tearing process with the $2 \mathrm{D}$ crosssection of the fracture process zone highlighting various stages during a monotonically increasing mode I loading ((1)-(4)). It is assumed that extensive crack growth has taken place such that the cut-out cross-section is located at a distance away from the crack initiation region which holds the transient history. The $2 \mathrm{D}$ section has the initial width, $W_{0}$, corresponding to the plate thickness, and the total height, $H_{0}$, is sufficiently large to engulf the thinning region that develops in the plate. The thinning spans approximately the region denoted by $h_{0}$, and complete elastic unloading above and below this domain takes place once the thinning initiates. Throughout all of the analyses, the initial aspect ratio of the domain is kept constant such that: $H_{0} / W_{0}=4$ and $h_{0} / W_{0}=2$. The loading is applied on the top and bottom edges of the $2 \mathrm{D}$ section. Here, monotonically increased by prescribing boundary displacements along the $x_{2}$-direction to mimic the far-field mode I loading condition.

The grey domains in Fig. 1(b), located above and below the region of interest, is taken to be composed of a homogeneous $J_{2}$ flow material (to reduce the computational cost), while only the fracture process zone is enriched by discrete and randomly distributed void nucleation sites (representing second phase particles). Figure 1(b) shows one realization of the model with the number of nucleation sites being $N_{p}=50$, while all nucleation sites share the same size of; $R_{p} / L_{e}=$ 15. Each nucleation site is modeled by prescribing a Gaussian bell distribution of the amount of damage that can nucleate (see Table 1 for the material parameters, and Osovski et al., 2015; Morgeneyer et al., 2016; Srivastava et al., 2017, for related studies). The nucleation sites are circular in shape with an initial radius of $R_{p} / L_{e}$, where $L_{e}$ is the edge length of the initially square-shaped finite elements used in the fracture process zone (see Section 3 for the details on the finite element model). The damage-related microstructure is created such that no nucleation sites overlap for $R_{p} / L_{e} \leq 9$ nor can they intersect the boundaries of the cross-section (for all values of $R_{p} / L_{e}$ considered). A homogeneously distributed background porosity is moreover allowed to nucleate in the domain surrounding the discrete nucleation sites (also governed by the Gurson model, see Section 3) to allow the crack to propagate between the nucleation sites. The nucleation parameters of the background porosity is kept small such that the crack path is not affected by the presence of the background porosity. Both types of nucleating voids are governed by strain-controlled nucleation (see Section 3). In this way, the model setup allows the size, $R_{p} / L_{e}$ (with $R_{p} / L_{e} \in[3,6,9,15,18]$ ), the number, $N_{p}$ (with $N_{p} \in[10,25,50,75,100]$ ), and the distribution of second phase particles to enter the prediction of the cohesive zone parameters suited for tearing of thin plates. Consider a $1 \mathrm{~mm}$ thick metal plate and the interval of size and number of void nucleation sites corresponds to inclusion sizes in the range $R_{p}=3.75 \mu \mathrm{m}-22.5 \mu \mathrm{m}$ and area fraction of $0.022-7.95 \%$. All combinations of the damage-related microstructure parameters are considered for three realizations of the random distribution of discrete nucleation sites. Throughout, the plate material is assumed to be non-porous initially before the deformation and with the mechanical properties summarized in Table 1.

\subsection{Cohesive traction-separation relation}

The material separation process, here governed by the micro-mechanics leading to failure, is typically imple- 


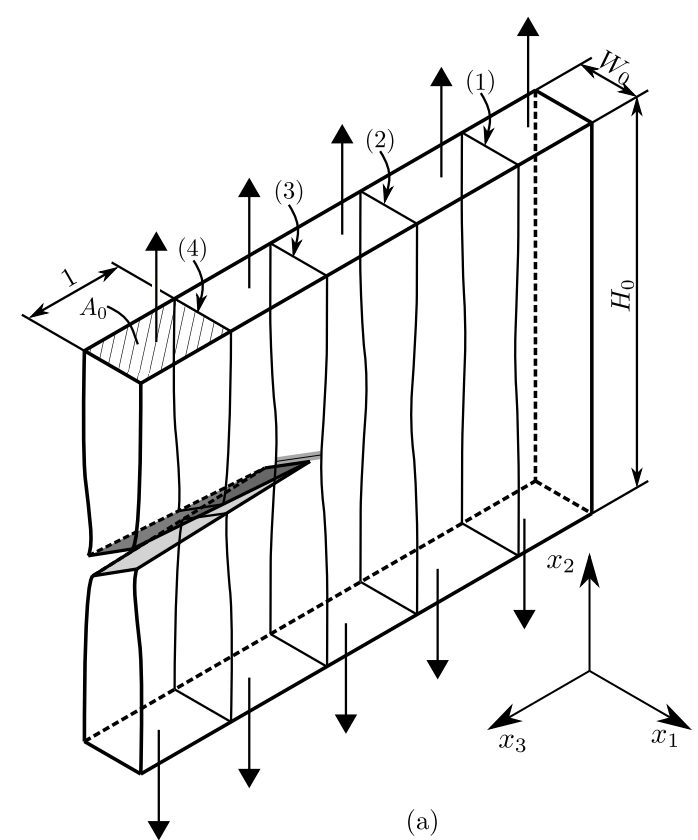

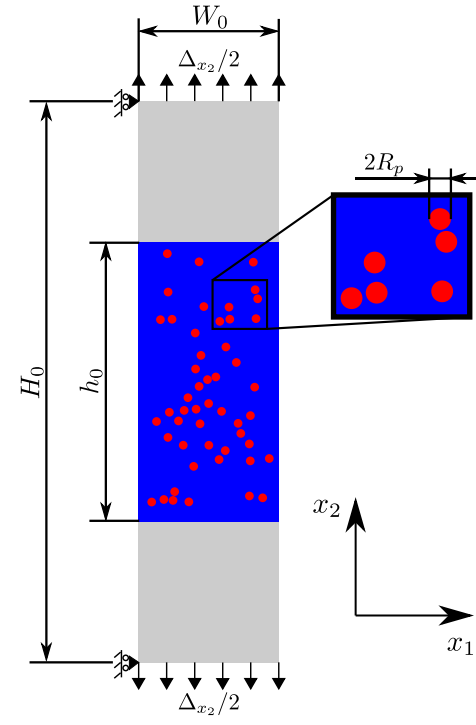

(b)

Fig. 1 (a) Schematic of the plate tearing process with different locations of the 2D plane strain cross-sections. (b) One realization of a plate with $N_{p}=50$ and $R_{p} / L_{e}=15$. The fracture process zone with discrete nucleation sites, highlighted in blue, has the height of $h_{0} / W_{0}=2$ and the height of the total domain is $H_{0} / W_{0}=4$. Prescribed boundary conditions are applied at the top/bottom boundaries. The left upper and lower nodes are constrained in the $x_{1}$-direction to prevent the two parts from moving freely after separation.

Table 1 Material properties

\begin{tabular}{|c|c|c|}
\hline Parameters & Notation & Value \\
\hline Density & $\rho$ & $2700 \mathrm{~kg} / \mathrm{m}^{3}$ \\
\hline Young's modulus & $E$ & $70 \mathrm{GPa}$ \\
\hline Poisson's ratio & $\nu$ & 0.3 \\
\hline Yield stress & $\sigma_{0}$ & $300 \mathrm{MPa}$ \\
\hline Strain hardening exponent & $N$ & 0.1 \\
\hline Gurson fitting parameters & $q_{1}, q_{2}, q_{3}$ & $1.5,1.0,2.25$ \\
\hline $\begin{array}{l}\text { Void volume fraction that } \\
\text { can potentially nucleate }\end{array}$ & $f_{N}^{n s}, f_{N}^{m}$ & $8 \cdot 10^{-3}, 1 \cdot 10^{-5}$ \\
\hline Mean strain for nucleation & $\varepsilon_{N}^{n s}, \varepsilon_{N}^{m}$ & $1 \cdot 10^{-2}$ \\
\hline $\begin{array}{l}\text { Standard deviation for } \\
\text { mean strain } \\
\text { Critical void volume }\end{array}$ & $s_{N}^{n s}, s_{N}^{m}$ & $(1 / 3) \cdot 10^{-2}$ \\
\hline fraction & $f_{C}^{n s}, f_{C}^{m}$ & $1 \cdot 10^{-2}$ \\
\hline fracture & $f_{F}^{n s}, f_{F}^{m}$ & $5 \cdot 10^{-2}$ \\
\hline
\end{tabular}

The superscripts "ns" refers to the "void nucleation sites", and " $\mathrm{m}$ " to the "matrix" material surrounding the nucleation sites

mented into a cohesive element through a traction-separation relation when dealing with large-scale shell element based models. In the case of plate tearing, the traction-separation relation holds the information about the separation after the peak force is attained and material softening is initiated, covering extensive thinning, secondary localization, and complete loss of load-carrying capacity (fracture). The adopted 2D modeling framework allows approximating this response by extracting the force- displacement curves for the cross-section considered. By relating the simulation response to a tri-linear tractionseparation relation, it is possible to identify: i) the peak force $\left(F_{\text {peak }}\right)$, i.e., the maximum force reached for the cross-section. This essentially coincides with the prediction of the Considère criterion (see the discussion in Nielsen and Hutchinson, 2012). The peak force is extracted at $\Delta_{x_{2}} / H_{0}=N$, with $N$ being the strain hardening exponent in the matrix material (the Considère strain) to facilitate a comparison basis between the various material configurations under investigation in this study. The choice was made due to difficulties in determining the peak force from a nearly flat response curve subject to minor fluctuations. ii) The point of secondary localization $\left(F_{\text {sec.loc. }}, \Delta_{\text {sec.loc. }}\right)$ is considered where the cross-section shows a great loss in load-carrying capacity. This is quantified as the point where the slope on the normalized curves takes the value of $\leq-12$ (a value estimated to match all calculations performed), and both the force and separation is recorded at that point. iii) The cohesive energy $\left(\Gamma_{0}\right)$ is determined as the area underneath the force-displacement curve extracted for the cross-section. It is worth to mention that these key parameters unambiguously define the tri-linear cohesive relation. The tri-linear relation, thereby, starts out with a nearly vertical inlet from zero traction and zero separation until the peak force is reached, with almost zero separation. The inlet is followed by a linear 
part that connects the peak force to the point of the secondary localization, after which the force drops to zero at some final separation set by the cohesive energy (see Fig. 2).

\section{Material and finite element model}

\subsection{Gurson material model}

The material in the fracture process zone of the $2 \mathrm{D}$ cross-section is assumed to be governed by the GursonTvergaard-Needleman (GTN) material model. The yield surface for the GTN material model is as follows:

$\Phi=\left(\frac{\sigma_{e}}{\sigma_{M}}\right)^{2}+2 q_{1} f^{*} \cosh \left(\frac{3 q_{2}}{2} \frac{\sigma_{m}}{\sigma_{M}}\right)-\left(1+q_{3}\left(f^{*}\right)^{2}\right)$

where $\sigma_{e}=\sqrt{3 s_{i j} s_{i j} / 2}$ is the effective macroscopic von Mises stress with $s_{i j}$ being the deviatoric part of the Cauchy stress tensor, $\sigma_{M}$ is the instantaneous yield stress of the fully dense matrix material, $\sigma_{m}=\sigma_{k k} / 3$ is the mean stress, $f^{*}$ is the effective void volume fraction, and $q_{1}, q_{2}$, and $q_{3}$ are fitting parameters introduced by Tvergaard (1981). The effective void volume fraction, $f^{*}$, takes into account the coalescence criterion based on a critical void volume fraction, $f_{C}$, and a final void volume fraction, $f_{F}$. When $f=f_{C}$ is reached, the effective void volume fraction accelerates in the following manner:

$f^{*}= \begin{cases}f & , \text { for } f \leq f_{C} \\ f_{C}+\frac{\bar{f}_{F}-f_{C}}{f_{F}-f_{C}}\left(f-f_{C}\right) & , \text { for } f>f_{C}\end{cases}$

with $\bar{f}_{F}=\left(q_{1}+\sqrt{q_{1}^{2}-q_{3}}\right) / q_{3}$, which reduces to $\bar{f}_{F}=$ $1 / q_{1}$ when $q_{3}=q_{1}^{2}$.

The total void volume fraction, $f$, evolves due to contributions from nucleation and growth of voids: $\dot{f}=$ $\dot{f}_{\text {nucl }}+\dot{f}_{\text {growth }}$. Nucleation of voids is strain-controlled such that $\dot{f}_{\text {nucl }}$ is given as:

$\dot{f}_{\text {nucl }}=\frac{f_{N}}{s_{N} \sqrt{2 \pi}} \exp \left[-\frac{1}{2}\left(\frac{\varepsilon_{M}^{p l}-\varepsilon_{N}}{s_{N}}\right)^{2}\right] \dot{\varepsilon}_{M}^{p l}$

where $\varepsilon_{M}^{p l}$ is the microscopic equivalent plastic strain in the matrix material, $\varepsilon_{N}$ is the microscopic mean strain for nucleation, $s_{N}$ is the standard deviation, and $f_{N}$ is the void volume fraction that can potentially nucleate. The void growth contribution is controlled by the change in plastic strains following: $\dot{f}_{\text {growth }}=(1-f) \dot{\varepsilon}_{k k}^{p l}$.

The matrix material follows the uni-axial stressstrain behavior:

$\sigma= \begin{cases}E \varepsilon & \text { for } \varepsilon<\varepsilon_{0} \\ \sigma_{0}\left(\frac{\varepsilon}{\varepsilon_{0}}\right)^{N} & \text { for } \varepsilon \geq \varepsilon_{0}\end{cases}$ where $E$ is the Young's modulus, $\varepsilon_{0}$ the initial yield strain, and $N$ the strain hardening exponent. All material parameter values are listed in Table 1.

\subsection{Finite element model}

The finite element model is generated in Abaqus/Explicit and is schematically shown in Fig. 1(b). The element type chosen to discretize the fracture process zone is the bilinear element type CPE4R with four nodes, reduced integration, governed by the assumption of plane strain. The fracture process zone in the middle of the $2 \mathrm{D}$ cross-section consists of initially square-shaped elements with an edge length of $L_{e} / W_{0}=1 / 800$ in the undeformed configuration (along the $x_{1}$-direction). The top and bottom regions, where the material is governed by $J_{2}$ flow theory, are discretized freely (i.e., by using both quadrilateral CPE4R and three-node linear CPE3 elements; see ABAQUS (2016) for detailed element properties). Thereby, the element size increases from the finely meshed fracture process zone towards the top and bottom boundaries. The finite element simulations are carried out in a dynamic framework but the loading is assumed to be quasi-static by controlling the deformation rate and ensuring the kinetic energy to be much lower than $10 \%$ of the total energy in the system. The CPU time for each simulation is less than 24 hours when performed on four central processing units in parallel on an HP Z420 workstation.

\section{Results: Cohesive zone parameters}

Figure 3 depicts the force-displacement curves for a wide span of plate materials with very different configurations of the damage-related microstructure. The displacement is the total elongation of the $2 \mathrm{D}$ crosssection normalized with the height $H_{0}$, and the force is calculated as the sum of the nodal forces, along $x_{2}$, on the top boundary $\left(\sum_{i}^{n} F_{i}\right)$ normalized with the initial yield stress and the initial surface area in the $x_{1}-x_{3^{-}}$ plane (see Fig. 1). In one end of the spectrum of materials investigated here is the case with a few small nucleation sites $\left(N_{p}=10\right.$ and $\left.R_{p} / L_{e}=3\right)$ that withstand severe plastic deformation even after the peak force is attained (yielding large cohesive energy). In contrast, a material with little to none post-peak ductility, due to strong interactions between many large nucleation sites, stands in the other end $\left(N_{p}=100\right.$ and $\left.R_{p} / L_{e}=18\right)$. Between these two extremes is a well of configurations that display intermediate post-peak ductility where the micro-mechanics governing localization and failure cannot be tied to a specific mechanism (see discussion in 


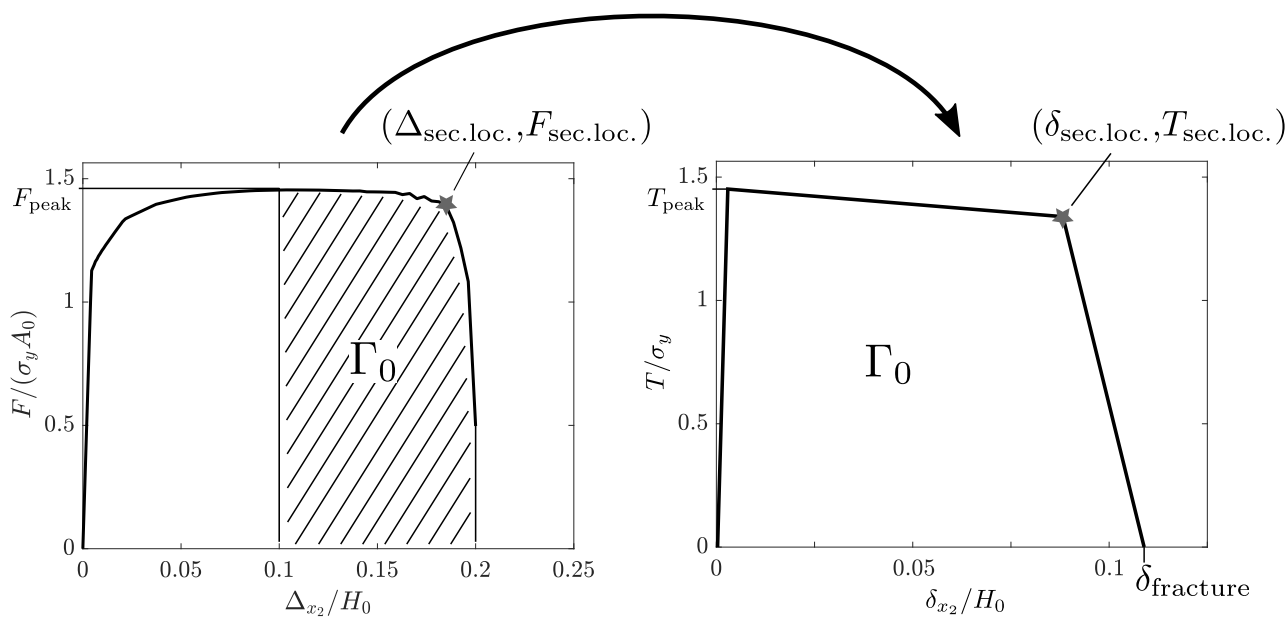

Fig. 2 Cohesive energy is defined from peak force to fracture (left figure). This is transferred to a cohesive traction-separation relation (right figure) controlling the behavior of the cohesive element. Here, $T$ is traction and $\delta$ is separation. The appearance of the relation is governed by the peak force, $F_{\text {peak }}$, and cohesive energy, $\Gamma_{0}$.

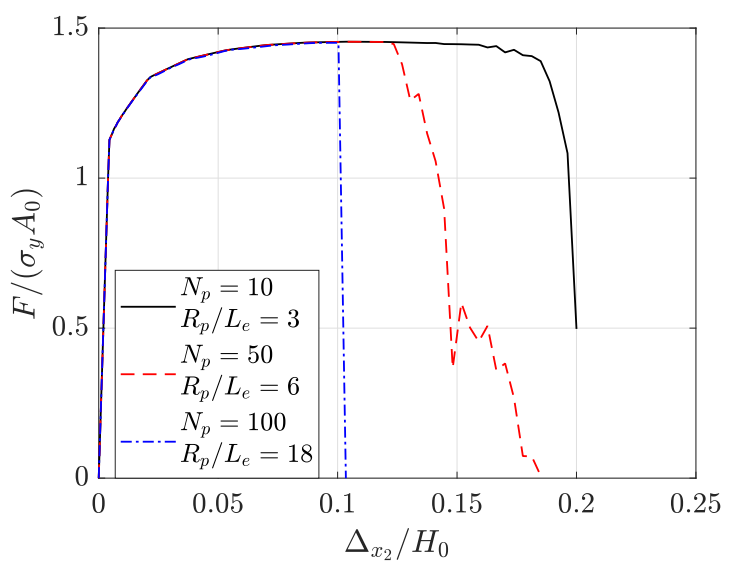

Fig. 3 Representative normalized force-displacement curves for various values of number $\left(N_{p}\right)$ and size $\left(R_{p} / L_{e}\right)$ of void nucleation sites.

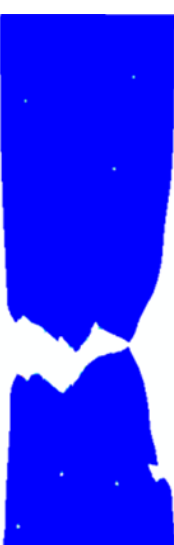

(a)

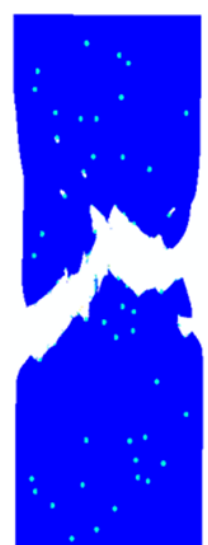

(b)

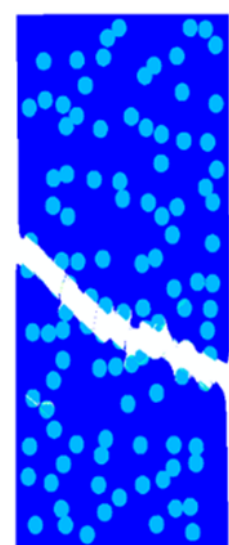

(c)
Fig. 4 Fracture surface morphology for three parameter sets corresponding to the ones shown in Fig. 3: (a) $N_{p}=10$, $R_{p} / L_{e}=3$, (b) $N_{p}=50, R_{p} / L_{e}=6$, and (c) $N_{p}=100$ $R_{p} / L_{e}=18$.

\subsection{The peak force}

Figure 5 shows the peak force for a fixed size of the nucleation sites versus the number of sites (Fig. 5(a)) and for a fixed number of sites versus size (Fig. 5(b)). It is clear that the mean peak force is essentially unaffected by the discrete event of void nucleation, and the largest decrease in the peak force is on the order of $0.2 \%$ between the various material configurations. The lack of variation is due to the interaction of nucleation sites being tied to the plastic straining that follows from the post-localization deformation (thinning), while the material largely responds as if it is homogeneous prior to the thinning (also justifying the modeling assumption in Section 2). In fact, comparing the model prediction to that of the Considère condition for a non-porous hoheterogeneous nucleation events during plate tearing. 
mogeneous cross-section subject to plane strain tension, given by; $T_{\max } / \sigma_{y}=2 / \sqrt{3}\left(2 N E /\left(\sqrt{3} \sigma_{y}\right)\right)^{N} \exp ^{-N}$, yields nearly coinciding predictions. The difference is on the order of $0.1 \%$ (based on the average value predicted), with the numerical model predicting higher values. This deviation is assigned to small inertia effects associated with the use of an explicit dynamic formulation. Nonetheless, it is seen from Fig. 5 that the mean peak force decreases as the number of nucleation sites increases while keeping the size of the nucleation site fixed and that the same holds when increasing the size while keeping the number of sites fixed. The largest decrease in mean peak force is predicted in the cases with many large nucleation sites. Three realizations for the random distribution are considered for each microstructure configuration (giving a total of 75 simulations), allowing both a mean and a standard deviation for the model predictions to enter into the results (see Fig. 5). Despite the standard deviation being of substantial dispersion for the largest nucleation sites considered, the results in Fig. 5 clearly demonstrate statistical evidence for a lower peak force when either increasing the number or size of the nucleation sites, although the variation is insignificant.

\subsection{The cohesive energy}

The trend for the cohesive energy is much more pronounced when compared to that of the peak force predictions as the model shows a significant drop in the mean energy going into material separation when increasing either the number or size of the nucleation sites. For example, the mean cohesive energy drops from $\Gamma_{0} /\left(\sigma_{y} W_{0}\right) \approx 0.258$ to 0.035 , when increasing the number of sites from 10 to 100, while keeping the size constant at $R_{p} / L_{e}=18$ (the largest sites considered). The rather low cohesive energy predicted for such large nucleation sites is tied to an early interaction between sites which allows localization across the entire cross-section to take place shortly after the peak force is attained. As seen in Fig. 4(c), this results in a limited thinning of the plate. Tekoğlu and Nielsen (2019) categorized the interaction between the individual void nucleation sites into three different mechanisms: (I) local $\pm 45^{\circ}$ shear bands near the void nucleation sites interact to form a global localization, (II) new shear bands are created through "void sheeting" where neighboring voids interact, and III neighboring void nucleation sites merge by internal necking of the ligament connecting them (see Figs. 6 and 12 of Tekoğlu and Nielsen (2019) for contour plots showing the three mechanisms and, additionally, the discussions in Bron et al., 2004; Buljac et al., 2018).

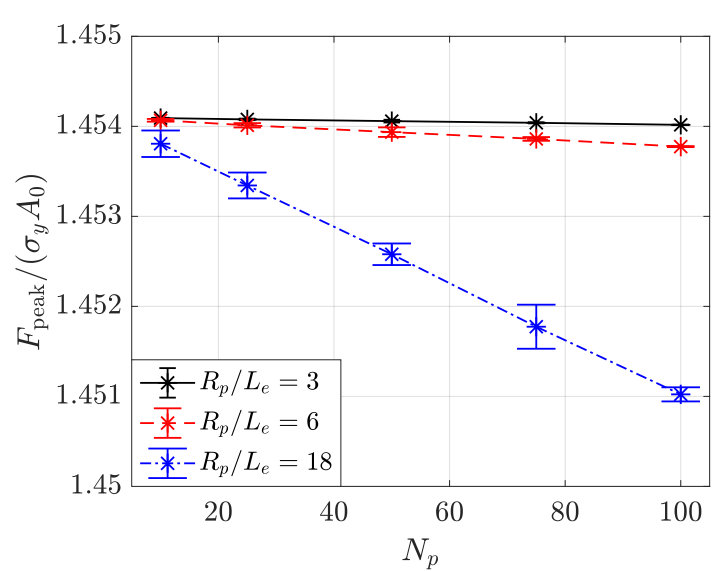

(a)

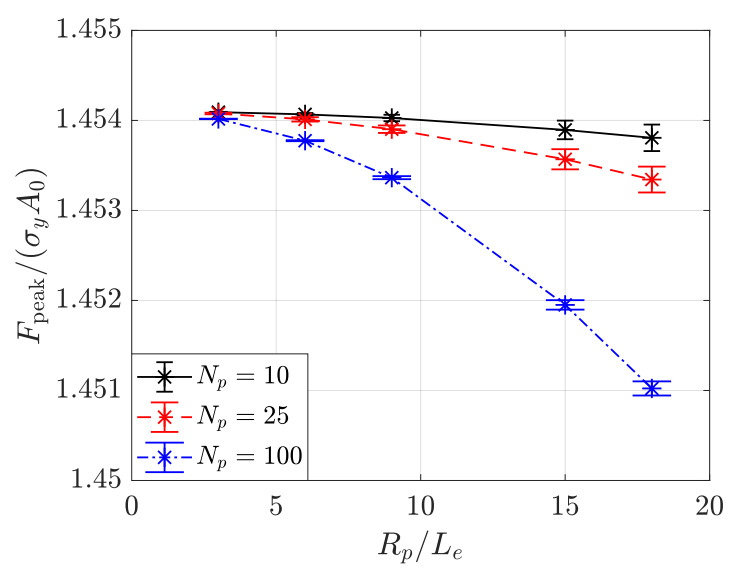

(b)

Fig. 5 Peak force as a function of (a) number $\left(N_{p}\right)$ and (b) size $\left(R_{p} / L_{e}\right)$ of void nucleation sites. The interval of confidence shown is $3 \sigma$, with the standard deviation being $\sigma$. The Considère force for a corresponding, non-porous, homogeneous cross-section is: $F_{\text {Considère }} /\left(\sigma_{y} A_{0}\right)=1.452$.

The predicted drop in the mean cohesive energy is associated with the shift from failure by mechanism (II) to failure by mechanism (I). The void sheeting mechanism (mechanism III) is prevailing in the case of a few large nucleation sites, which leads to a slant fracture at an angle lower than $45^{\circ}$, whereas mechanism (I) sets in when the number of nucleation sites is large, allowing existing $45^{\circ}$ local shear band that emanate from the individual sites to coalesce and form global localization at an angle of $45^{\circ}$ across the entire plate thickness (see Fig. 4(c) and Fig. 9 in Tekoğlu and Nielsen, 2019). Consistent slant fracture is predicted for all realizations of the random distribution with large nucleation sites, which, in turn, results in the rather narrow interval of confidence (using $3 \sigma$ with $\sigma$ being the standard deviation) seen in Fig. 6(a). In contrast, the interval of confidence is somewhat larger for the case of small nucle- 


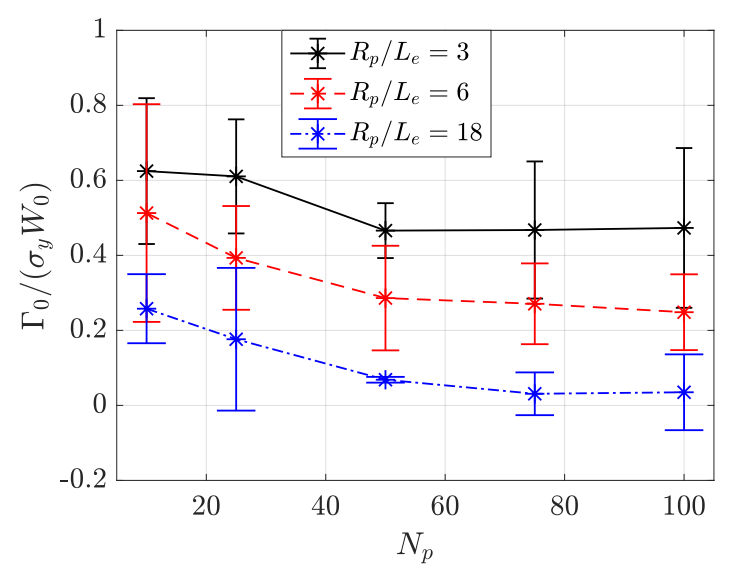

(a)

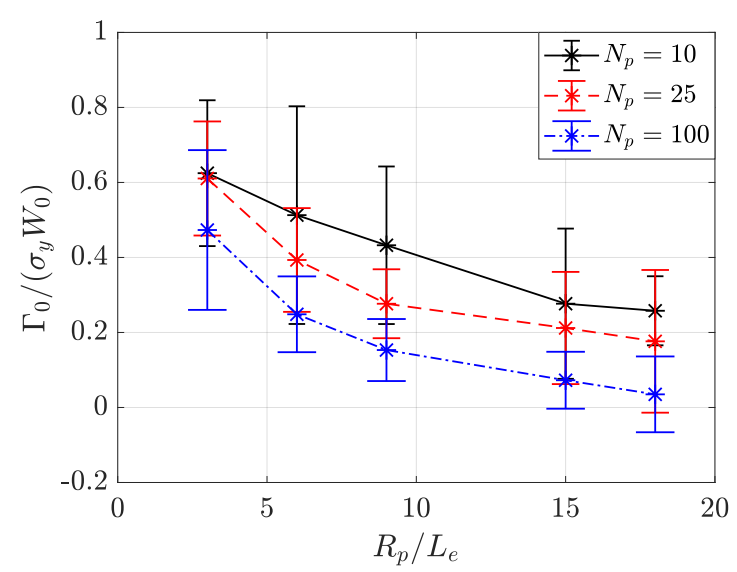

(b)

Fig. 6 Cohesive energy as a function of (a) number $\left(N_{p}\right)$ and (b) size $\left(R_{p} / L_{e}\right)$ of void nucleation sites. The interval of confidence shown is $3 \sigma$, with the standard deviation being $\sigma$.

ation sites $\left(R_{p} / L_{e}=3\right)$. This has to do with the fracture surface morphology being much less well-defined and with, essentially, all of the three mechanisms identified by Tekoğlu and Nielsen (2019) coming into play. In these cases, the linking of nucleation sites requires pronounced thinning of the plate, yielding a higher mean cohesive energy due to severe plastic deformation when the crack opens. It is important to emphasize that for this case where $R_{p} / L_{e}=3$, despite that the mean cohesive energy displays a drop for an increasing number of nucleation sites, the interval of confidence is too wide to form a statistical basis for a general conclusion. In addition, it is worth to mention that the cohesive energy cannot be negative as indicated by the interval of confidence in Fig. 6, e.g. for $R_{p} / L_{e}=18$. The indicated negative energy is merely an outcome of the statistical analysis.

Keeping a fixed number of nucleation sites and increasing their size (see Fig. 6(b)) also yield a consistent drop in the mean cohesive energy within the parameter interval considered. This is despite overlapping intervals of confidence (using $3 \sigma$ ). Thus, a plate material with a few small nucleation sites displays the highest mean cohesive energy, while the energy is lowest in the case of many large sites. Recall that this shift in the mean cohesive energy is tied strongly to the thinning of the plate prior to fracture and, thus, the overall strain hardening capacity - also discussed in Nielsen and Hutchinson (2012) for homogeneous porous plate materials. The change in the plate thinning is realized by consulting Fig. 11 in Tekoğlu and Nielsen (2019). In fact, a shift in the failure mechanism clearly occurs when increasing the size of the nucleation site while keeping the distribution and number of sites fixed. Small nucleation sites give rise to severe thinning and failure by a mixture of mechanisms (I) through (III), while large sites primarily link up by mechanism (I). For an intermediate size of the nucleation sites, a mixture of mechanisms (I) and (II) is obtained. In addition, comparable energy levels are observed when consulting the predicted level for the mean cohesive energy in Fig. 6 to that of the homogeneous porous plate material considered in Nielsen and Hutchinson (2012). A direct comparison between the two studies, however, should be made with care as the damage in the fracture process zone is very different. The highest value for the cohesive energy in Fig. 6 is predicted for the case with; $R_{p} / L_{e}=3$ and $N_{p}=10$, which is slightly higher than for the homogeneous porous material in Nielsen and Hutchinson (2012). However, the general decrease in energy level, when accounting for nucleation sites, underlines that great care must be taken for plate materials that either diverges from a homogeneous initial configuration (e.g., in thin plates) or are prone to significant discrete nucleation events.

\subsection{Secondary localization and loss of load-carrying capacity}

The displacement at the onset of the secondary localization, where the material rapidly loses its load-carrying capacity, is reported in Fig. 7. The displacement at this point displays a similar dependency on the damagerelated microstructure as the cohesive energy. This is not surprising as the area under the force-displacement curve, which defines the cohesive energy, is strongly related to the displacement at the secondary localization. Despite the somewhat large standard deviations, especially for the smallest nucleation sites, the displacement at the onset of the secondary localization increases for the diminishing size of the nucleation sites - for all cases 
considered - as this leads to higher post-peak ductility, severe diffuse thinning, and postponed secondary localization. Essentially, smaller particles give rise to a higher overall strain hardening capacity. On the other hand, the case with many large sites shows a low overall strain hardening capacity with secondary localization and rupture shortly after the peak force is attained. The force level at the onset of the secondary localization is depicted in Fig. 8 and it is found to be much less influenced by the damage-related microstructure. The interval of confidence is wide for nearly all configurations, but the tendency is that the force drops with decreasing size of the nucleation sites (see Fig. 8(a)). In fact, the shift in post-peak ductility with respect to changes to the damage-related microstructure is clearly brought out in Fig. 8. There exists a threshold on the number of sites (this is dependent on the size of the nucleation sites) where the force at the secondary localization essentially takes the value of the peak force attained at the primary localization set by the plate thinning. Thus, the secondary localization occurs simultaneously with, or shortly after, the primary localization (a related discussion on microscopic versus macroscopic localization can be found in Tekoğlu et al., 2015; Tekoğlu and Nielsen, 2019).

The fairly large dispersion in the predictions for the force at the onset of the secondary localization, when considering different random distributions, is highlighted by Fig. 9. Here showing the curves for three realizations of randomly distributed nucleation sites when $R_{p} / L_{e}=$ 3 and $N_{p}=100$. A close-up of the load-displacement curves reveals fluctuations in the curve at the point (automatically) identified with the onset of the secondary localization (see also Section 2.2).

\section{Concluding remarks}

The present work demonstrates the connection between the damage-related microstructure and the parameters going into a cohesive traction-separation relation. Consequently, a link is created between the underlying micromechanics and the otherwise phenomenological parameters. Plate materials with randomly distributed discrete void nucleation sites, of various sizes and numbers, have been analyzed, and the main results of the analyses are summarized below.

(i) The peak force is virtually unaffected by changing the number, size, and distribution of the discrete nucleation sites (see Fig. 5). The main effect on the peak force relates to the material softening originating from the nucleated damage. The peak force predicted for the various heterogeneous materials

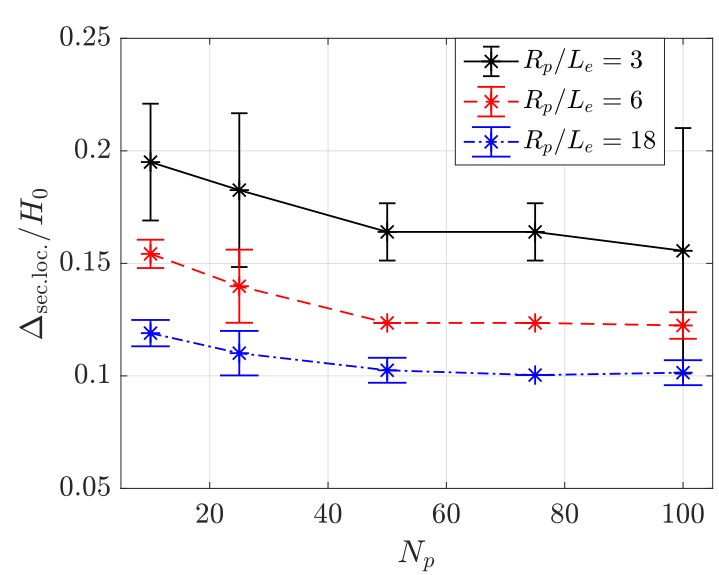

(a)

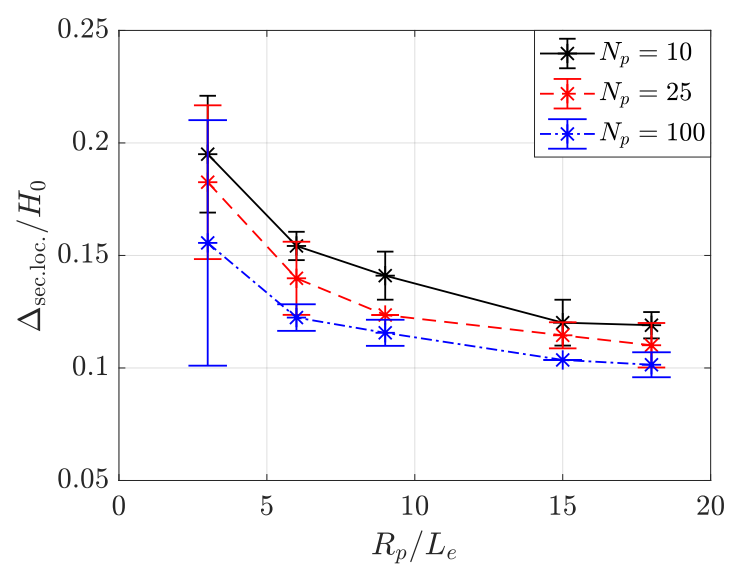

(b)

Fig. 7 Strain at secondary localization as a function of (a) number $\left(N_{p}\right)$ and (b) size $\left(R_{p} / L_{e}\right)$ of void nucleation sites. The interval of confidence shown is $3 \sigma$, with the standard deviation being $\sigma$.

all display low standard deviations yielding a firm statistical basis for this conclusion. Moreover, the model predictions for the heterogeneous materials closely agree with similar calculations for homogeneous porous plate materials (see Nielsen and Hutchinson (2012)), meaning that the Considère criterion for plane strain tension accurately approximates the peak force. Thus, accounting for the damage-related microstructure in modeling large-scale plate structures, e.g., using shell elements, requires no special attention regarding the constitutive modeling of the material that surrounds the cohesive zone.

(ii) The cohesive energy depends highly on both the number and size of the discrete nucleation sites. An increase in either one causes a decrease in the mean cohesive energy which, in turn, leads to the reduction in the overall strain hardening capacity of the plate material. Despite the partially overlapping in- 


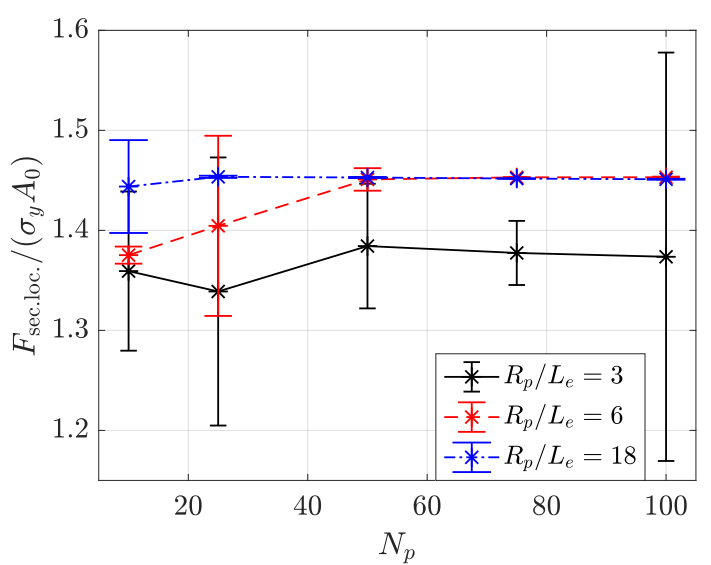

(a)

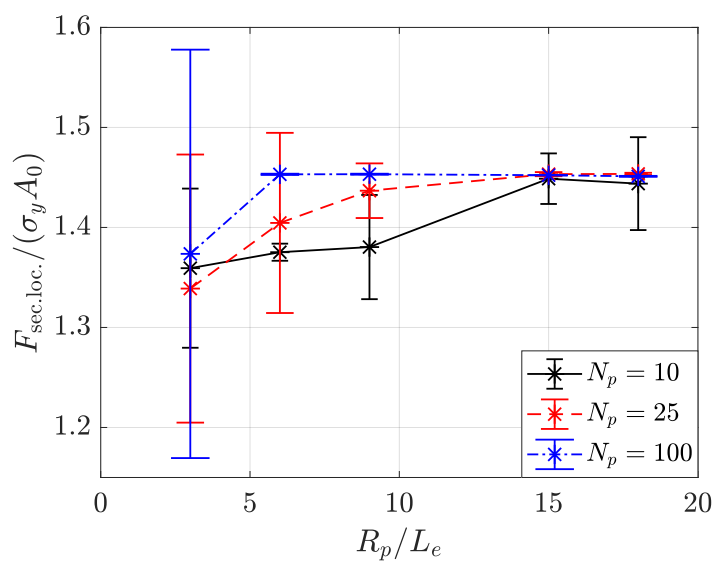

(b)

Fig. 8 Force at secondary localization as a function of (a) number $\left(N_{p}\right)$ and (b) size $\left(R_{p} / L_{e}\right)$ of void nucleation sites. The interval of confidence shown is $3 \sigma$, with the standard deviation being $\sigma$. The Considère force for a corresponding, nonporous, homogeneous cross-section is: $F_{\text {Considère }} /\left(\sigma_{y} A_{0}\right)=$ 1.452 .

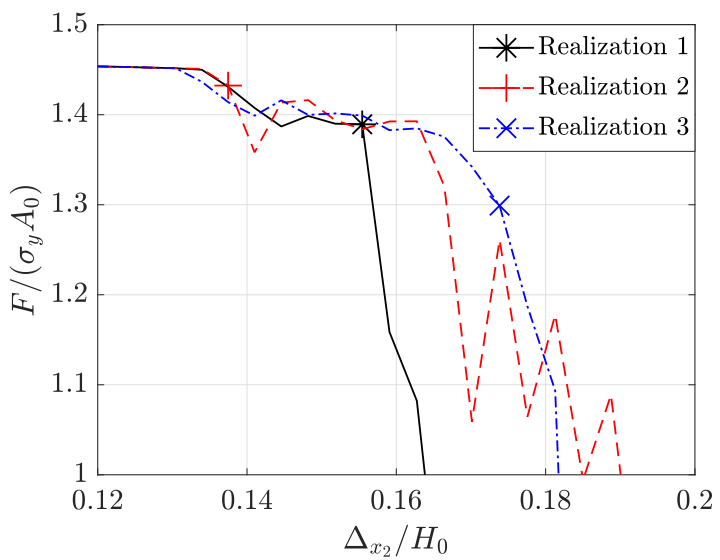

Fig. 9 Zoom in on the overall response approaching fracture where the asterisks denote the onset of secondary localization according to the procedure employed. Model parameter set here is $N_{p}=100$ and $R_{p} / L_{e}=3$. terval of statistical confidence, the trends are clear from Fig. 6. The strong dependency of the cohesive energy on the number and size of the nucleation sites suggests that variations in the tearing mechanism can develop as the crack propagates in large plates. Thus, manufacturers must ensure tight control over the size and number of nucleation sites to achieve a plate material with a uniform fracture surface morphology resulting from large-scale plate tearing. Along the same lines, the modeling of large-scale plate tearing must take into account any variation in the damage-related microstructures, occurring along the crack path, to accurately predict failure.

The standard deviation for the cohesive energy displays a rather large dispersion and, thus, an attempt to reduce this dispersion has been made by including more realizations. By adding two more realizations, giving a total of five, for $R_{p} / L_{e}=6$ and all values of $N_{p}$ did, however, not provide the desired reduction. Three realizations for each material configuration are, therefore, used throughout the present study.

(iii) The onset of secondary localization, in terms of the displacement, follows the trend for the cohesive energy and displays a substantial drop when increasing the number and/or size of the nucleation sites (see Fig. 7). The force at the secondary localization is, on the other hand, only slightly lower than the peak force and displays a weak dependency on the size and number of nucleation sites (see Fig. 8). Interestingly, there exists a threshold on the number of sites (depending on their size) for which the secondary localization coincides with, or occurs shortly after, the primary localization (see Fig. 8 or Tekoğlu et al., 2015, for a related discussion). Thus, information on the force at the secondary localization (even an estimate) is suggested to be used in combination with the cohesive energy when creating the microstructure dependent traction-separation relation in Fig. 2 for a given plate material.

Nielsen and Hutchinson (2012) separated the cohesive energy into two parts, one originating from the diffuse thinning and the other related to the secondary localization into shear bands. A similar distinction between energies has not been possible for the current setup as the point of final separation displays a significant standard deviation (Fig. 3 indicates this large scatter). Nonetheless, the study demonstrates that the parameters going into the cohesive traction-separation relation require to be tuned when the damage-related microstructure diverges from a homogeneous configuration. 
Acknowledgements The authors gratefully acknowledge the financial support by TÜBİTAK (Project No: 315M133). RGA is financially supported by the Department of Mechanical Engineering at the Technical University of Denmark in the project "Advancing Numerical Analysis of Large Scale Crack Propagation in Plate Structures". KLN is financially supported by Independent Research Fund Denmark in the project "Advanced Damage Models with InTrinsic Size Effects" (Grant no: DFF-7017-00121).

\section{References}

ABAQUS (2016) Abaqus documentation collection, Version 2016, Providence, RI: Dassault Systèmes

Andersen R, Woelke P, Nielsen K (2018) Cohesive traction-separation relations for plate tearing under mixed mode loading. Eng Frac Mech 71:199-209

Andersen R, Felter CL, Nielsen KL (2019) Micromechanics based cohesive zone modeling of full scale ductile plate tearing: From initiation to steadystate. International Journal of Solids and Structures 160:265-275

Bron F, Besson J, Pineau A (2004) Ductile rupture in thin sheets of two grades of 2024 aluminum alloy. Materials Science and Engineering a 380(1):356-364, DOI 10.1016/j.msea.2004.04.008

Buljac A, Helfen L, Hild F, Morgeneyer TF (2018) Effect of void arrangement on ductile damage mechanisms in nodular graphite cast iron: In situ 3d measurements. Engineering Fracture Mechanics 192:242261, DOI 10.1016/j.engfracmech.2018.01.008

Cornec A, Scheider I, Schwalbe K (2003) On the practical application of the cohesive model. Engineering Fracture Mechanics 70(14):1963-1987, DOI 10.1016/S0013-7944(03)00134-6

Gologanu M, Leblond J, Perrin G, Devaux J (1997) Recent extensions of Gurson's model for porous ductile metals. In Continuum Micromechanics, SpringerVerlag, Berlin pp 61-106

Gurson A (1977) Continuum theory of ductile rupture by void nucleation and growth - part I: yield criteria and flow rules for porous ductile media. ASME J Eng Mater Technol 99:2-15

Morgeneyer T, Taillandier-Thomas T, Buljac A, Helfen L, Hild F (2016) On strain and damage interactions during tearing: 3D in situ measurements and simulations for a ductile alloy (AA2139-T3). J Mech Phys Solids 96:550-571

Nielsen K, Hutchinson J (2012) Cohesive tractionseparation laws for tearing of ductile metal plates. Int J Imp Eng 48:15-23

Noell P, Carroll J, Boyce B (2018) The mechanisms of ductile rupture. Acta Mater 161:83-98
Osovski S, Srivastava A, Ponson L, Bouchaud E, Tvergaard V, Ravi-Chandar K, Needleman A (2015) The effect of loading rate on ductile fracture toughness and fracture surface roughness. J Mech Phys Solids $76: 20-46$

Osovski S, Needleman A, Srivastava A (2019) Intergranular fracture prediction and microstructure design. Int J Frac 216:135-148

Pardoen T, Hachez F, Marchioni B, Blyth P, Atkins A (2004) Mode I fracture of sheet metal. J Mech Phys Solids 52:423-452

Srivastava A, Ponson L, Osovski S, Bouchaud E, Tvergaard V, Needleman A (2014) Effect of inclusion density on ductile fracture toughness and roughness. J Mech Phys Solids 63:62 - 79

Srivastava A, Osovski S, Needleman A (2017) Engineering the crack path by controlling the microstructure. J Mech Phys Solids 100:1-20

Tekoğlu C, Nielsen K (2019) Effect of damage-related microstructural parameters on plate tearing at steady state. European J Mech A/Solids 77:103818

Tekoğlu C, Hutchinson J, Pardoen T (2015) On localization and void coalescence as a precursor to ductile fracture. Phil Trans R Soc A373

Tvergaard V (1981) Influence of voids on shear band instabilities under plane strain condition. Int J Fract Mech 17:389-407

Tvergaard V, Hutchinson J (2002) Two mechanisms of ductile fracture: Void by void growth versus multiple void interaction. Int J Solid and Struct 39:3581-3597

Woelke PB, Shields MD, Hutchinson JW (2015) Cohesive zone modeling and calibration for Mode I tearing of large ductile plates. Eng Frac Mech pp 293-305

Zhao L, Ding L, Soete J, Idrissi H, Kerckhofs G, Simar A (2019) Fostering crack deviation via local internal stresses in $\mathrm{Al} / \mathrm{NiTi}$ composites and its correlation with fracture toughness. Composites Part A 126:105617 